\title{
VISUAL AID FOR THE DISABLED
}

\section{HARSH SHAH $^{1}$, SIDDHANT BHATT $^{2}$ \& SAHIL MAHENDRA MANGAONKAR ${ }^{3}$}

${ }^{1,2}$ Department of Electronics Engineering , D.J Sanghvi College of Engineering, Mumbai, India

${ }^{3}$ Department of Electronics and Telecommunication Engineering, D.J Sanghvi College of Engineering, Mumbai, India

ABSTRACT
Blindness, or severe sight impairment, can affect people of any age. An estimated253 million people are visually
challenged and out of those, approximately 36 million people are blind. The multitude of difficulties faced by them on
a day to day basis is immense. Owing to the advancementsin science and technology, a lot of research has been done to
find ways to improve the lifestyle ofthe partially-sighted and blind people. Reading and recognition devices could make
smartphones, tablets and smart glasses into indispensable aids for the visually impaired.
The aim of the project is to provide assistance to visually impaired people. We aim to provide navigation
guidance for the blind and hence aim to do our bit to improve their lives. The product first receives input from a
identify and classify the objects detected. We then identify which object out of the total objects is the closest to the
observer in order to give the necessary feedback actions. After these processing steps the objects that are closest to the
person or that might have the potential to harm them are detected and their positions are informed to the person using
a speaker. This enables the person to become aware of their surroundings and thus act accordingly.
KEYWORDS: Autonomous Navigation, Yolo Architecture, Image Processing Algorithms, Computer Vision
Raspberry Pi, Convolutional Neural Networks

Received: Aug 27, 2020; Accepted: Sep 17, 2020; Published: Sep 30, 2020; Paper Id.: IJECIERDDEC20202

\section{INTRODUCTION}

Autonomous navigation is extremely important for people with visual impairment. While walking canes and guide dogs have been used since time immemorial, these lack the capability of covering a long range for obstacle detection. With the rapid development and advance in technology, there has been a significant progress in the use of headgears for assistance. This feat in technology has expanded and opened up new horizons for intelligent navigation capabilities. Different approaches for obstacle detection using ultrasound, laser and RADAR have already been employed. Our aim in this project is to employ the visual assistance using image processing algorithms.

We have further used 3 approaches in order to find out which object is the closest to the observer using image processing. Instead of implementing the distance measurement using sensors we have decided to use image processing since it promises more accurate results, owing to the costs of sensors. On the hardware side, the project is implemented on a Raspberry Pi connected locally to a laptop via the Wi-Fi protocol.

The objectives of the project can be divided as follows:

- Obstacle detection and classification 
- Localization and perception of objects

- Audio output of feedback action

\section{SYSTEM MODEL/ARCHITECTURE}

The system we aim to implement is a blind and vision assistant. The functioning of the system is divided into several parts and hence a large number of algorithms from Machine Learning to computer vision and other optimization algorithms are employed. Our first aim is to implement an object detection system by using image processing. Object detection is done by employing Convolutional Neural Networks and other deep learning algorithms like YOLO. On detecting the object, the next task of the system is to differentiate between a moving and a non-moving object, living and non-living thing, etc. We use a predefined dataset called as COCO which has 80 labels from person, dog, and bus to lamppost, toothbrush and cell phone. Such an extensive dataset helps us to classify and recognize the objects with greater precision and efficiency. YOLO is a state-of-the-art object detection algorithm that is incredibly fast and accurate

\section{Convolutional Neural Networks:}

In neural networks, Convolutional Neural Network is one of the main categories to do images recognition, images classifications. CNNs are general used to extracts features from the image. Problems like face recognition and object detection can be performed using neural networks but the number of parameters used and the processing speed required is tremendously large. These drawbacks are tackled by using convolutional neural networks.

Technically, deep learning CNN models to train and test, each input image will pass it through a series of convolution layers with filters (Kernels), Pooling, fully connected layers (FC) and apply Softmax function to classify an object with probabilistic values between 0 and 1 . The below figure is a complete flow of CNN to process an input image and classifies the objects based on values.

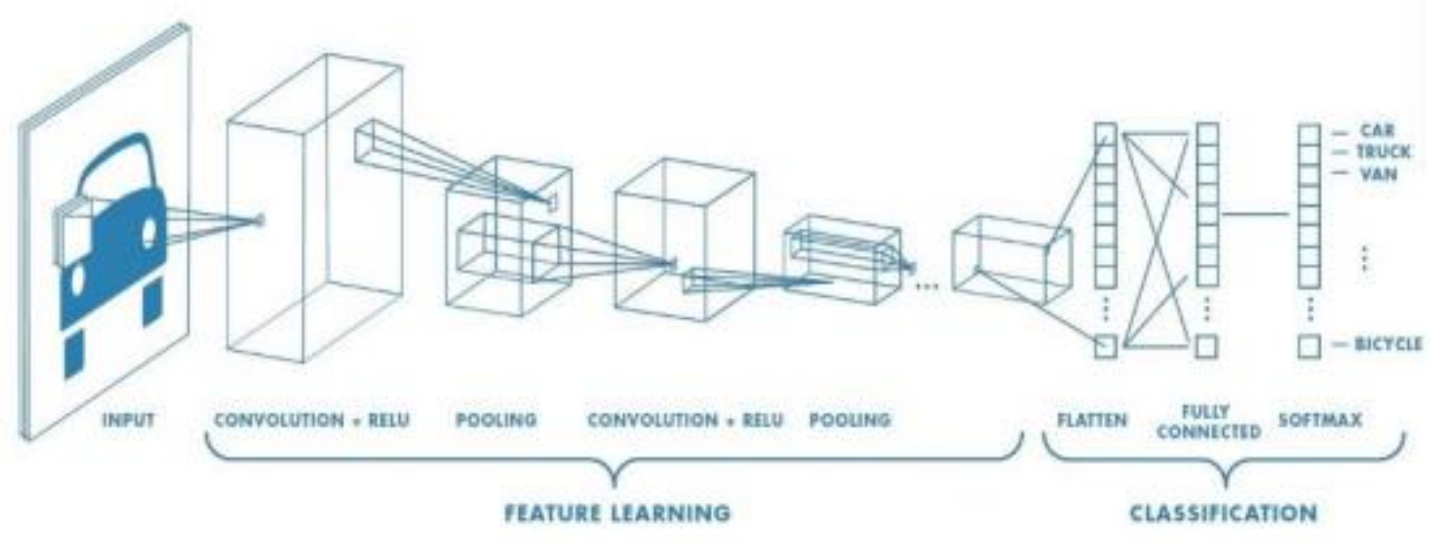

Figure 1

\section{Convolution Layer:}

Convolution is the first layer to extract features from an input image. Convolution preserves the relationship between pixels by learning image features using small squares of input data. It is a mathematical operation that takes two inputs such as image matrix and a filter or kernel. Convolution of an image with different filters can perform operations such as edge detection, blur and sharpen by applying filters. The below example shows various convolution image after applying 
different types of filters.

There are two types of CNN:

- Valid CNN - Dimensions of the output matrix is less than the input matrix.

- Same CNN - Padding of input matrix is done to keep the dimensions of input and output same.

\section{Working of Yolo:}

- The algorithm first divides the image into a grid of SxS blocks.

- Each block predicts $\mathrm{N}$ possible objects using image classification and localization and places a bounding box on it, calculates the level of certainty (confidence) of each box. Thus, $\mathrm{S} x \mathrm{~S}$ x N boxes are created and calculated.

- The majority of such boxes will have a very low level of certainty; thus, the algorithm deletes those boxes whose certainty level is lower than the set threshold level.

- The remaining boxes are then passed through a "non-max suppression" that deletes objects that have been detected more than once (duplicate objects) and leaves only the most exact one of them.

- For object detection, we used the MS COCO pre-trained dataset. This dataset was trained using the DarkNet code base and can classify up to 80 different objects from dogs and cats to balls, laptops, cars, etc. Such an extensive dataset helps us to classify and recognize the objects with greater precision and efficiency.

\section{Yolo Architecture:}

The detection layer consists of 24 convolutional layers followed by 2 fully connected layers. Alternate $1 \mathrm{x} 1$ convolutional layers reduce the features space from preceding layers. The final output of the network is a 7x7x30 tensor of predictions.

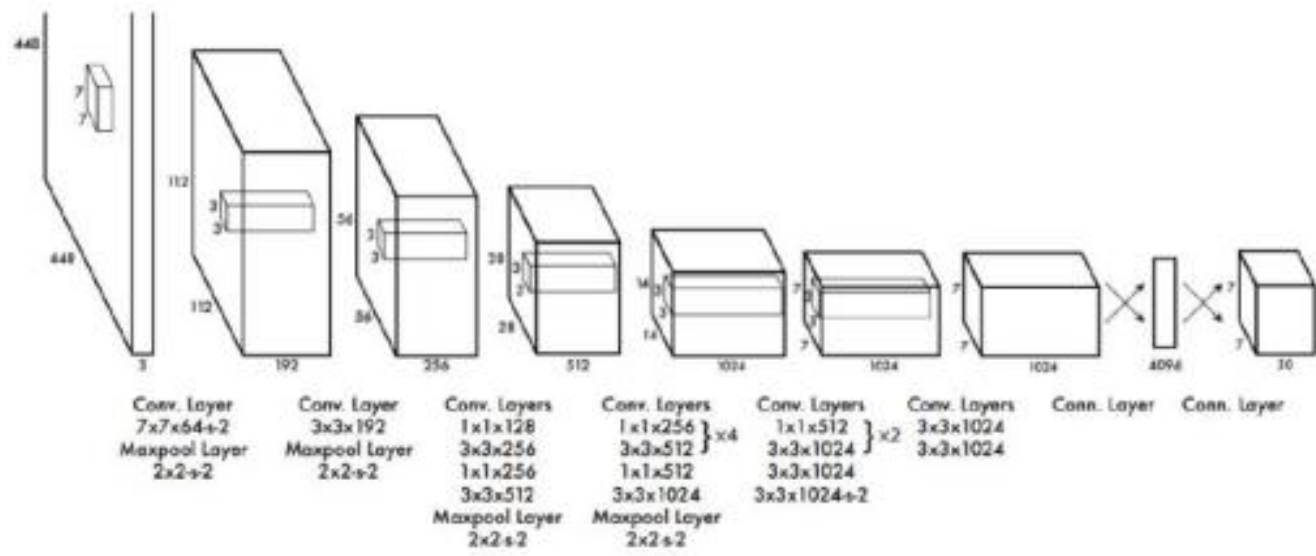

Figure 2

\section{Intersection over Union:}

Intersection over Union calculates the ratio of the intersecting area of the actual bounding box and the predicted bounding box for an object to the union of the areas of the bounding boxes.

$I o U=$ Areaofintersection /Areaofunion 
If IoU is greater than 0.5 , the prediction is good enough. The value 0.5 is taken arbitrarily and can be changed according to the requirement. The higher the value of the threshold, the better the predictions become.

\section{Non-Max Suppression:}

This algorithm is used to remove the multiple bounding boxes formed around the same object. First, the boxes with confidences less than the threshold set are removed. Out of the remaining boxes, the one with the highest confidence value is selected. The IoU value for all the remaining boxes for the same object is then calculated. If the IoU value for any such box is greater than the pre decided threshold value, such boxes are then suppressed. These steps are then repeated, until all the detected objects are bound by only a single box.

\section{Prediction Vector:}

The input image is divided into SxS grid cells. Each grid cell predicts B bounding boxes and C class probabilities. There are 5 components in the bounding box prediction: $\{\mathrm{x}, \mathrm{y}, \mathrm{w}, \mathrm{h}$, confidence $\}$. Here, $(\mathrm{x}, \mathrm{y})$ represents the coordinates of the centre pixel of the object with respect to the grid cell location. These coordinates have been normalized to [0,1]. (w,h) provide the width and height, i.e. the dimensions of the box which have also been normalized to [0,1] relative to the image size. The confidence prediction is defined as the IoU between the predicted box and the ground truth box. If no object exists in a cell, the confidence is zero.

Each grid vector also predicts $\mathrm{C}$ conditional class probabilities. Regardless of the number of bounding boxes B, only one set of class probabilities per grid cell is calculated. During the test time, the conditional class probabilities and the individual box predictions are multiplied to produce a class-specific confidence values for each box. These values define the probability of the class appearing in the box as well as how well the predicted bounding box fits the object.

Yolo Vs. Tiny Yolo:

The YOLO algorithm requires large processing power and more memory. The main reason for this is large model size and less inference speed. This makes it unsuitable for its use on embedded devices like the Raspberry Pi. Thus, to overcome this, we used Tiny YOLO algorithm.

Tiny YOLO is a smaller version of the YOLOv2 model and is made up of 9 convolutional layers and 6 maxpooling layers. The model accepts the input image of the shape $416 \times 416 \times 3$. The output produced is a $125 \times 13 \times 13$ tensor with the image being divided into $13 \times 13$ grid cells. Each grid corresponds to 125 channels, made from 5 bounding boxes that are predicted by the grid cell and the 25 data elements that describe each bounding box.

The major advantage of this model are:

- It has a smaller model size, thus requires lesser memory.

- Instead of using TensorFlow on the backend, it uses TensorFlow Lite, which has been developed to enable on device machine learning inference with a small binary size and low latency.

Table 1 


\begin{tabular}{|c|c|c|c|c|c|}
\hline Neural Network & Input Resolution & Iterations & Avg loss & average loU(\%) & mAP (\%) \\
\hline Tiny Yolo V3 & $416 \times 416$ & 42000 & 0.1983 & $45.59 \%$ & $61.18 \%$ \\
\hline Tiny Yolo V3 & $608 \times 608$ & 21700 & 0.3469 & $46.39 \%$ & $61.29 \%$ \\
\hline Tiny Yolo V3 & $832 \times 832$ & 55200 & 0.2311 & $48.69 \%$ & $56.77 \%$ \\
\hline Yolo V3 & $416 \times 416$ & 19800 & 0.1945 & $0.00 \%$ & $0.00 \%$ \\
\hline Yolo V/3 & $608 \times 608$ & 2900 & 0.71 & $42.63 \%$ & $23.46 \%$ \\
\hline Yolo V3 & $832 \times 832$ & 5600 & 0.3324 & $38.77 \%$ & $41.20 \%$ \\
\hline
\end{tabular}

The next phase of the project included the calculation of distance of identified objects from the user. For calculating distance through image processing, we found out 3 approaches namely,

- Distance measurement using similarity of triangles

- $\quad$ Point Triangulation Method

- $\quad$ Stereo Reconstruction using Disparity maps

Distance measurement is done using similarity of triangles method using only one camera. It is based on the geometric principle which states that the ratios of sides of similar triangles are equal. Here, the distance is measured by comparing the ratios of lengths of two triangles that are formed. This method has been explained in chapter 4 extensively. The triangulation method and stereo vision approach follow a similar set of principles and they use 2 cameras for finding the distance accurately. Since they use two cameras, camera calibration is required. Both the binocular distance measurement approaches are based on the concept of stereo vision.

\section{Camera Calibration:}

Cameras are often untuned and thus suffer from various image distortion problems. The camera that we used suffered from radial distortion and for it to be used, it had to be rectified.This is done by using a chess board. About 50 images of the chess board are acquired from different poses of the camera. The real-world distances between each block on the chess board is known. Then by matching the points on the chess board on all the images and by computing their differences , we were able to find the intrinsic and extrinsic parameters of the camera like camera matrix, projection matrix , rotation and translation vectors, etc.

\section{Disparity Maps:}

Disparity maps is defined as the apparent pixel difference or motion between a pair of stereo images. Such a map can be used to make an intensity map out of the measurements, which can be used to calculate the depth of objects in an image and thus find their distance from the camera centre. Disparity maps use the concept of stereo reconstruction and epipolar geometry to produce a single map with different pixel intensities that define different depths of objects in the scene. The objects closer to the camera will have a lighter hue and the objects that are far away will have a darker hue. 


\section{PROPOSED SYSTEM IMPLEMENTATION:}

\section{STEP 1: Data Acquisition}

Data in our system is in the form of images. A human eye sees an image or object and further perceives it in the brain after the optical neuron sends the signal to the brain. A similar concept is used and implemented in a computer, the image is broken down into a matrix of pixels and stored in the memory of a computer. The matrix pixels have a value depending on the type of colour spaces used, i.e. HSV (Hue Saturation Value), RGB (Red Green Blue) and Grayscale. In our implementation we have used RGB colour space in order to process them for recognition.

Due to constraints on the availability of large number of labelled dataset and limited computational power, we choose to use a pre-trained model of object recognition based on YOLO. While pre-training the model, it was found that the best results were obtained by taking input image by using RGB colour space. Since the test data should match the train data, the input that we are taking is also in RGB. The convolutional neural network kernel is slid over each individual channel to identify objects, if present in the frame.

\section{STEP 2: Object Recognition}

Object detection, in our project, is done by using computer vision for identifying objects' instances in images or videos. Object detection algorithms typically leverage machine learning or deep learning to produce meaningful results. When humans look at images or video, we can recognize and locate objects of interest within a matter of moments. The goal of object detection is to replicate this intelligence using a computer. Object detection uses a number of machine learning algorithms and the one which we have used is an advanced form of a neural network called as Convolutional Neural Networks which is used to implement the YOLO algorithm. Recognizing an object and identifying it is a very important step for our application since the feedback signal heavily depends on the type of obstacle in front of the human. In order to recognize the object, we need a labelled dataset which we obtain from a library called COCO. The COCO dataset has labelled data consisting of 80 distinct classes which map to a number of similar images and hence it proves to be effective in our application. Machine Learning algorithm of CNN is used to identify the object and map the features of the image to the corresponding features of the trained dataset.

Since the accuracy of the model is not $100 \%$, there are chances of false recognition i.e. the detected object is not mapped to the right class . To minimize this error, we calculated how confidently the model is recognizing the object. All the detections with confidence level below threshold are discarded at this stage. After trial and error, we found out the threshold to be 0.7 i.e. the model is $70 \%$ confident that the detected object is rightly recognized.

\section{STEP 3: Distance Measurement}

We are using camera for object detection purpose and using the same for calculating the distance will cut down on hardware cost.

The two inverter triangles formed by the actual height and distance (ABO), and the focal length of lens and the height in terms of pixels (DCO), are similar to each other. Thus, by property of similarity we can say that,

$$
\begin{aligned}
& F / h p=d / h \\
& \therefore d=F * h / h p
\end{aligned}
$$




\section{STEP 4: Region Identification:}

The image is split into three parts namely right, left and centre. The width of image is divided in 5 equal parts. The $\mathrm{x}$ coordinate and y-coordinate of the centre of detected object is used to locate the position of that object. If the centre of object in consideration is in the first part i.e. in between 0 and width/5, then the object is said to be towards the right of the user. Similarly, if the centre of detected object is in between pixels that range from width/4 and width, then the object is to the left of user. Otherwise, the detected objectis in front of the user.

\section{STEP 5: Feedback Signal:}

The processor and the algorithm, based on the obstacles and the distance, returns an output which tell us whether the obstacle near or far from the observer. At the same time, it returns a vocal output to the user as a feedback mechanism. This enables the user to understand the positions of objects detected in his/her nearby vicinity.

After detecting objects in the frame and calculating their distances, only the objects that are in the close proximity of the user are passed on to the audio function. The speech output acts as the feedback for the user and helps him/her in perceiving the surrounding. The audio output specify which object is detected and where is it located (right, left or centre) with respect to the user.

In order to implement the same on a hardware platform, we used the library named festivals since pyttsx 3 could not support RPi platform. Raspberry Pi contains the "festivals" voice module package for producing voice outputs. The output device was connected to the $3.5 \mathrm{~mm}$ Audio jack available on the Raspberry pi for the voice output.

\section{STEP 6: Developing a Scalable Product:}

After testing the entire system on software in a high processing capability computer, we aim to make this system into a fully functional, scalable and cost-effective product in order to provide assistance to the visually disabled. The product will be used as an eyeglass by the disabled person and it will have a camera and a processor embedded into it in order to view the surroundings and process the output. It will also have an audio codec for speech output of the feedback reaction to be taken. As a part of the implementation of this future scope, we deployed a part of code on RPi and are currently working on making a finished product. The object detection model was also implemented on a Raspberry Pi 3B+ board. The model was able to detect and classify objects and produce audio output. Here, due to the limitation of lower processing power available, a different version of the YOLO algorithms (Tiny YOLO) was implemented.

\section{CONCLUSIONS}

Since there is no technological and cost-effective product solution for the visually disabled, our project aims to fill the gap for this demand. We aim to serve a social cause in order to increase the efficiency and inclusion of the disabled people in the society. Through the use of various machine learning and computer vision algorithms, we have successfully implemented the vision aid project. By giving a voice output, we aim to provide a real-time feedback so that no additional help is needed in order to perceive the surroundings. By employing various algorithms and approaches for depth sensing, we get a deeper understanding in the field of depth perception and monocular vision. We further understand the drawbacks of a sensor- based approach and the possibilities of innovation in computer vision for perceiving surroundings. 


\section{ACKNOWLEDGEMENTS}

Weare grateful toour MentorProf.Chandrashekhar Beral for providing technical assistance.His mentoring and total guidance helped us at every stage for this paper. And this paper would not have possible without his immensehelp. We thank our colleagues who provided advice and guidance that greatly contributed to the development of the paper. Also the way everyone found our mistakes and corrected themwas of great help to us.

\section{REFERENCES}

1. Samartha Koharwal, Samer Bani Awwad, Aparna Vyakaranam "Navigation System for Blind - Third Eye" International Journal of Innovative Technology and Exploring Engineering (IJITEE) ISSN: 2278-3075, Volume-8 Issue-5 March, 2019

2. Adrian Rosebrock Find distance from camera to object/marker using Python and OpenCV.

3. https://www.pyimagesearch.com/2015/01/19/find-distance-camera-objectmarker-using-python-opencv/

4. Joseph Redmon, Santosh Divvala, Ross Girshick, Ali Farhadi "You Only Look Once: Unified, Real-Time Object Detection" University of Washington, Allen Institute for AI, Facebook AI Research

5. DRISTI: Dynamic Ranging by Image Segmentation and Terrain Imaging- Samanth S Mokshagundam, Suhas G, Suhas T Shanbhogue, Suraj $S$

1. 